\title{
Erratum
}

\section{Protective Role of Gut Commensal Microbes against Intestinal Infections}

\author{
My Young Yoon ${ }^{1,3}$, Keehoon Lee $^{1,2}$, and Sang Sun Yoon ${ }^{1,2,3 *}$ \\ ${ }^{1}$ Department of Microbiology and Immunology, ${ }^{2}$ Brain Korea 21 Project for Medical Sciences, \\ ${ }^{3}$ Institute for Immunology and Immunological Diseases, Yonsei University College of Medicine, \\ Seoul 120-752, Republic of Korea
}

In the article by Yoon et al. published in Journal of Microbiology 2014; 52, 983-989. First author name should be changed as Mi Young Yoon 